\section{Antibiotic yields anxiolytic}

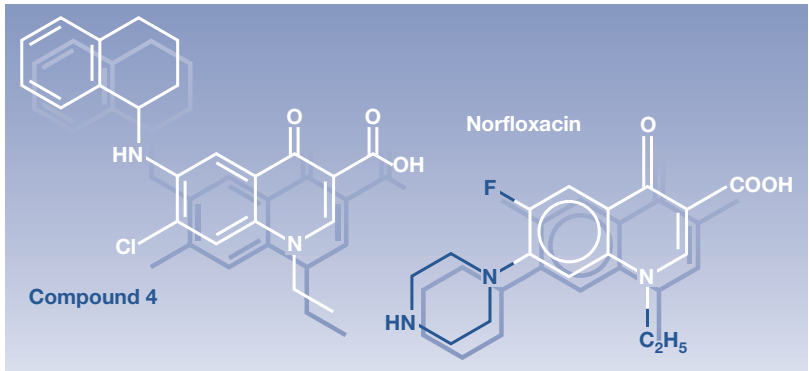

Benzodiazepines, such as diazepam, are commonly prescribed to control anxiety, and act by enhancing the action of the neurotransmitter GABA $(\gamma$-aminobutyric acid) at the brain
GABA $_{\mathrm{A}}$ receptor complex (GRC). But drugs of this class have sedative side effects, an undesirable feature that has spurred a search for alternatives by Kelvin Gee and colleagues. In a Nature Medicine advanced online publication, the team describe a first-in-class anxiolytic that is free of sedative side effects.

The search strategy devised by Gee et al. was based on the hypothesis that mitigation of side effects might be achieved by targeting non-benzodiazepine-binding sites of the GRC. Compounds related to the fluoroquinolone antibiotic norfloxacin were chosen as screening candidates for two reasons: first, a small proportion of patients taking this antibiotic experience anxiety as a side effect; second, norfloxacin is known to antagonize $\mathrm{GABA}_{\mathrm{A}}$ receptors at an undefined site. So, chemical modification of norfloxacin could generate compounds with the opposite, GABAenhancing anxiolytic effect.
In a standard assay for GABA modulation, several norfloxacinrelated chemotypes inhibited the binding of the convulsant TBPS to the GRC. Analysis of the most active product - dubbed compound 4 showed that this compound does not act directly on the GRC-binding sites for TBPS, norfloxacin, GABA or benzodiazepines.

To confirm the GABA-modulatory action of compound 4, electrophysiological studies were conducted on HEK293 cells expressing different human GRC subtypes. Treatment with compound 4 enhanced GABAmediated currents in a dose-dependent manner, but only in those cells that expressed the $\alpha_{2}$ subunit of the receptor. Failure of the benzodiazepine binding-site antagonist flumazenil to block this potentiation confirmed that compound 4 exerts its effect by binding to a non-benzodiazepine site. So structural manipulation of norfloxacin produced a compound that binds to

\title{
Shifting the cut of APP
}

In addition to their role in preventing pain and inflammation, some nonsteroidal antiinflammatory drugs (NSAIDs), such as sulindac sulphide, ibuprofen and indomethacin, have the unexpected benefit of reducing the risk of developing Alzheimer's disease. But why only certain NSAIDs seem to protect against Alzheimer's disease is a perplexing question. According to a recent study published in Science, the answer, it seems, depends on the interaction of these drugs with the small GTP-binding protein (G protein) Rho, and Rho's influence on what forms of amyloid- $\beta(A \beta)$ are produced.

The $A \beta$ peptide is generated when the amyloid precursor protein (APP) is cut by $\gamma$-secretase, and because $\gamma$-secretase can cut APP at various points, $A \beta$ peptides of different lengths are produced $(38,40$ or 42 amino acids). But it is the 42 -amino-acid variant, $A \beta_{42}$, that is predominant in the brain plaques that are a pathogenic hallmark of Alzheimer's disease. Previous research had suggested that certain NSAIDs protect against Alzheimer's disease by reducing the amount of $A \beta_{42}$, through an unknown mechanism that was independent of their antiinflammatory activity. As NSAIDs have also been shown to inhibit signalling by the small $\mathrm{G}$ protein Ras, Zhou and colleagues decided to investigate the effects of small G-protein signalling on the regulation of $A \beta_{42}$.

They first showed that increasing the activity of small $\mathrm{G}$ proteins in cultured cells selectively increased the concentration of $\mathrm{A} \beta_{42}$ over total $\mathrm{A} \beta$, an increase that could be completely blocked by the NSAIDs sulindac sulphide and ibuprofen. This activity was pinpointed to Rho, which is involved in numerous cellular processes, including cell migration and survival, transcriptional regulation and vesicle transport.

In cultured cells, inhibition of the Rho effector Rho-associated kinase (Rock) by the compound Y-27632 reduced $A \beta_{42}$ levels in a concentration-dependent manner, and was also shown to block Rho-mediated increases in $\mathrm{A} \beta_{42}$, suggesting that Rock acts downstream of Rho in the regulation of $A \beta_{42}$. Furthermore, treating a transgenic mouse model of Alzheimer's disease with Y-27632 reduced brain levels of $A \beta_{42}$ and the ratio of $\mathrm{A} \beta_{42}$ to total $\mathrm{A} \beta$. So, the Rho-Rock pathway has an important role in regulating $A \beta_{42}$ levels, both in vitro and in vivo.

The authors then showed that the NSAIDs sulindac sulphide, ibuprofen and indomethacin inhibited Rho activity. By contrast, the NSAIDs naproxen, meloxicam, SC-560 and piroxicam, which are ineffective at lowering levels of $A \beta_{42}$, showed little effect on the activity of Rho. The $\mathrm{A} \beta_{42}$-lowering NSAIDs also increased the levels of $A \beta_{38}$, and the researchers showed that altering the activity of Rho and Rock also affects both $A \beta_{42}$ and $A \beta_{38}$ levels. So, they suggest that Rho-Rock signalling modulates the cleavage specificity of $\gamma$-secretase, and that NSAIDs therefore also alter the cutting activity of $\gamma$-secretase through their interaction with Rho.

The side effects of NSAIDs, which include gastrointestinal and renal toxicity, might preclude their long-term use to prevent Alzheimer's disease, but perhaps isolating the Rho-inhibitory activity of these drugs could lead to a novel approach to treat the disease.

Clare Ellis

(4) References and links ORIGINAL RESEARCH PAPER Zhou, Y. et al. Nonsteroidal anti-inflammatory drugs can lower amyloidogenic $A \beta_{42}$ by inhibiting Rho. Science 302, 1215-1217 (2003) FURTHER READING Weggen, $\mathrm{S}$. et al. A subset of NSAIDs lower amyloidogenic $A \beta_{42}$ independently of cyclooxygenase activity. Nature 414, 212-216 (2001) 
an alternative GRC site, but did this modification result in anxiolytic rather than anxiety-inducing effects?

In two rodent models of anxiety, treatment with compound 4 relieved anxiety-related behaviour. Notably, the magnitude of this effect was comparable to that afforded by diazepam. But the real breakthrough came from assessments of motor performance using the rotarod test: diazepam caused motor impairment whereas compound 4 did not, even at the maximal soluble dose. Compound 4 might therefore be at the forefront of a new approach to the design of robust anxiolytics with improved side-effect profiles.

Suzanne Farley

\section{(4) References and links} ORIGINAL RESEARCH PAPER

Johnstone, T. B. C. et al. Modifying quinolone antibiotics yields new anxiolytics. Nature Med. 30 Nov 2003 (doi: 10.1038/nm967) FURTHER READING Christopoulos, A. Allosteric binding sites on cell-surface receptors: novel targets for drug discovery. Nature Rev. Drug Discov. 1, 198-210 (2002)
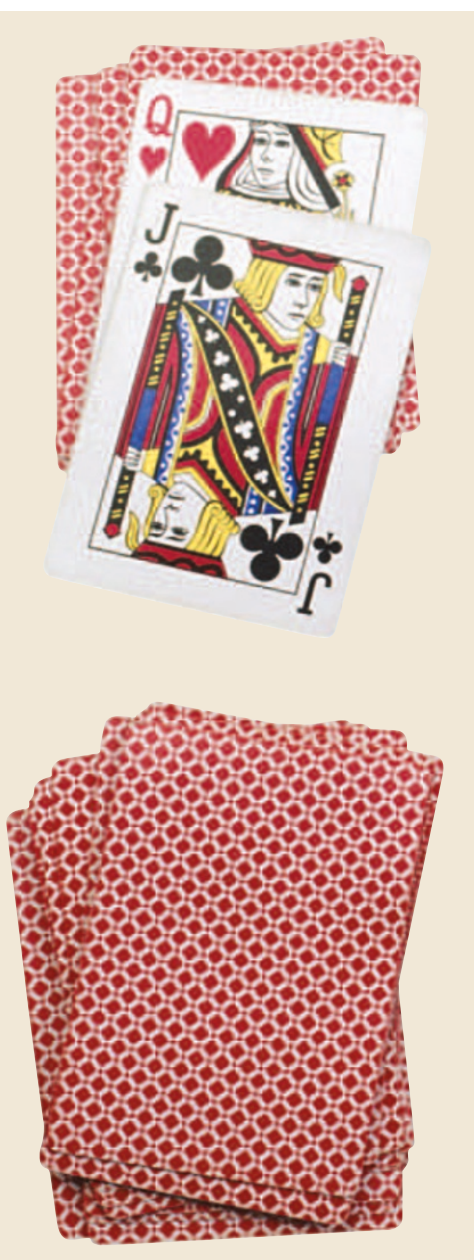

TARGET VALIDATION

\section{Of mice and men and rats...}

Knockout mice are now widely used to provide validation of the therapeutic potential of molecular targets - a strategy that is backed up by evidence that the knockout phenotypes for the targets of many leading drugs correlate well with known drug efficacy. Nevertheless, it is important to remember that this might not always be the case, as highlighted by a recent paper by Hirst et al. in Molecular Pharmacology, which demonstrates marked differences between the distribution and pharmacology of a particular serotonin receptor subtype in humans and rats, and that observed in mice.

Serotonin (5-hydroxytryptamine, 5-HT) is a neurotransmitter that exerts a wide variety of physiological and behavioural effects by acting on one or more of at least seven 5-HT receptor subtypes. The rat and human versions of the 5-HT ${ }_{6}$ receptor, which is a G-protein-coupled receptor, were cloned in the mid-1990s, and seem to show similar pharmacological properties and distribution in the brain. Since then, various studies have provided evidence that antagonists of the $5 \mathrm{HT}_{6}$ receptor might boost cholinergic neurotransmission and thereby ameliorate the cognitive impairments experienced by patients with dementia or schizophrenia, raising interest in this receptor as a drug target.

However, when Hirst and colleagues attempted to study the brain distribution of mouse 5- $\mathrm{HT}_{6}$ receptors using a radioligand known as $\left[{ }^{125} \mathrm{I}\right] \mathrm{SB}-258585$ that selectively labels the human and rat $5-\mathrm{HT}_{6}$ receptors, their experiments indicated that there was no specific binding of the radioligand. Prompted by these unexpected observations, they set out to compare the brain distribution of the $5-\mathrm{HT}_{6}$ receptor in mouse, rat and human brain by using the reversetranscriptase polymerase chain reaction. Indeed, they found that in the rat and human brain, $5-\mathrm{HT}_{6}$ receptors were expressed in several regions, particularly the striatum, but in the mouse there was far less expression of the 5- $\mathrm{HT}_{6}$ receptor in all brain regions examined, and no enrichment in the striatum.

The authors also carried out experiments to investigate the pharmacology of the $5-\mathrm{HT}_{6}$ receptor in mouse, rat and man using recombinant receptors. Binding studies showed that the affinity of a range of serotonergic agonists and antagonists at the mouse $5-\mathrm{HT}_{6}$ receptor differed considerably from that at the rat and human 5- $\mathrm{HT}_{6}$ receptors. Investigation of four amino acids identified from sequence alignments as potentially responsible for these differences using mutant $5-\mathrm{HT}_{6}$ receptors found that two residues - one in helix 5 and one in helix 6 accounted for the majority of the differences in pharmacology. Furthermore, computational studies of the receptor and ligands, which predicted that the binding pocket of the mouse $5-\mathrm{HT}_{6}$ receptor was different from the rat and human 5- $\mathrm{HT}_{6}$ receptors, were in excellent agreement with the results obtained from the experiments with the mutant receptors.

Overall, the findings of Hirst et al. show that the mouse $5-\mathrm{HT}_{6}$ receptor differs in many key respects from the $5-\mathrm{HT}_{6}$ receptor in rats and humans. In general, this suggests that before the phenotype of a mouse with a particular molecular target knocked out is accepted as validation of that target, or as a model of human disease, it would be wise to ascertain that the molecular target has a pharmacology and distribution in mice similar to that in humans.

Peter Kirkpatrick

(9) References and links

ORIGINAL RESEARCH PAPER Hirst, W. D. et al. Differences in the central nervous system distribution and pharmacology of the mouse 5-hydroxytryptamine-6 receptor compared with rat and human receptors investigated by radioligand binding, site-directed mutagenesis, and molecular modelling. Mol. Pharmacol. 64, 1295-1308 (2003)

FURTHER READING Zambrowicz, B. P. \& Sands, A. T. Knockouts model the 100 best-selling drugs - will they model the next 100 ? Nature Rev. Drug Discov. 2, 38-51 (2003)

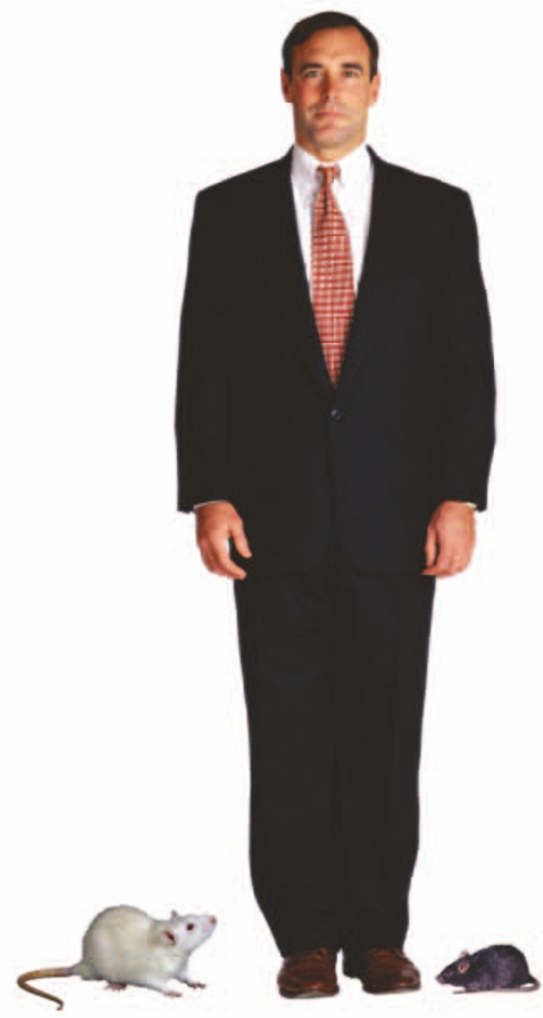

